\title{
Magnetohydrodynamic Effects on Insulating Bubbles and Inclusions in the Continuous Casting of Steel
}

\begin{abstract}
J.W. HAVERKORT and T.W.J. PEETERS
The magnetohydrodynamic effects associated with a magnetic field perpendicular to the movement of insulating inclusions or bubbles in a conducting liquid are investigated in this article. An increase in drag coefficient as a result of the presence of a magnetic field is argued to have a significant effect on their terminal rise velocity. Inside a continuous steel caster, this lower terminal velocity has a potentially negative effect on the removal rate of unwanted inclusions, degrading the steel quality. Simulations of an insulating rigid sphere moving in the presence of an electrical current show an electromagnetophoretic force per unit volume of $-\psi \mathbf{J} \times \mathbf{B}$, with a shape factor $\psi \approx 1.0$. Numerical fluid and dispersed gas phase simulations of the flow inside a submerged entry nozzle show that, because of this force, inhomogeneous magnetic fields can cause nonuniform gas distributions in accordance with a theoretical analysis. In particular, the magnetic field can be tailored to increase or decrease the amount of gas near the side walls.
\end{abstract}

DOI: $10.1007 / \mathrm{s} 11663-010-9415-\mathrm{z}$

(C) The Author(s) 2010. This article is published with open access at Springerlink.com

\section{INTRODUCTION}

MAGNETIC fields are often used in the processing of liquid metals to dampen unwanted fluid motion at free surfaces, control the degree of turbulence, or stir the liquid. Although electrically insulating inclusions or bubbles in a conducting fluid do not experience a direct Lorentz force, a magnetic field can still have a significant influence on their motion. Because the surrounding conducting liquid is affected by Lorentz forces, immersed insulating objects can experience noticeable magnetohydrodynamic effects indirectly. The most well known of these forces is the electromagnetophoretic force, which is also known as the electromagnetic buoyancy force or electromagnetic Archimedes force. ${ }^{[1]}$ This force acts as a reaction to the Lorentz force experienced by the fluid, similar to its gravitational counterpart. Some quantitative results regarding this important force can be found in References 2 and 3 . The electromagnetophoretic force is used frequently to separate nonmetallic inclusions from molten metals as a preprocessing step in the manufacturing of metals. This separation technique, which was practiced in the early 1970s in the former Soviet Union, ${ }^{[4]}$ took off in the West after a publication by Marty and Alemany. ${ }^{[5]}$ Occasionally, the electromagnetophoretic force is included explicitly in computational models; see Reference 6 for an example regarding rigid inclusions. As we will argue, the influence of the electromagnetophoretic force on the dynamics of a dispersed gas phase can also be significant.

J.W. HAVERKORT is with Delft University of Technology, Lorentzweg 1, 2628 CJ Delft, The Netherlands. Contact e-mail: J.W.Haverkort@cwi.nl T.W.J. PEETERS is with Tata Steel Research, Development \& Technology, 1970 CA IJmuiden, The Netherlands.

Manuscript submitted June 17, 2010.

Article published online August 12, 2010.
Another effect that received far less attention in the modelling of insulating immersions in liquid metals is the observation that in the presence of a magnetic field, the drag force on insulating objects increases. For rigid spherical objects, significant theoretical, ${ }^{[7-9]}$ experimental, ${ }^{[10-12]}$ and computational ${ }^{[13-15]}$ work has been reported. Recently, experiments with deformable bubbles have been performed ${ }^{[16,17]}$ showing that in this case, a magnetic field modifies the drag coefficient as well. For an applied magnetic field perpendicular to the bubble motion, a magnetohydrodynamically induced increase in drag coefficient similar to that of a rigid sphere has been reported. ${ }^{[16]}$ For bubble motion parallel to the magnetic field, however, the drag coefficient was found to decrease for bubbles larger than approximately $5 \mathrm{~mm}$. This effect could be attributed to the fact that fluid motion perpendicular to the bubble trajectory is, in this case, damped by the perpendicular magnetic field. This process causes the bubble to move in a straighter path, leading to a lower apparent drag force. Recent numerical simulations ${ }^{[18]}$ show that a perpendicular magnetic field acts to make a bubble more spherical, causing an increase in the drag force. A parallel magnetic field, however, gives the bubble a bullet-like shape, which leaves the drag coefficient almost unmodified.

\section{THEORETICAL CONSIDERATIONS}

In this section, we investigate the magnetohydrodynamic increase in drag force and the electromagnetophoretic force, which was discussed in the introduction, more quantitatively.

\section{A. Magnetohydrodynamically Modified Drag}

In the following, we will refer to both insulating solid inclusions and bubbles as immersions. In the description 
of these objects, we will neglect any inertial or memory effects. The equation of motion for the slip velocity $u_{s}$ of immersions relative to the fluid motion is in this case dominated primarily by the vertical force balance between drag and the difference between buoyancy and gravity,

$$
\frac{1}{2} \rho C_{D}\left(\frac{1}{4} \pi D^{2}\right) u_{s}^{2}=\left(\frac{1}{6} \pi D^{3}\right) g\left(\rho-\rho_{i}\right)
$$

where $\rho$ and $\rho_{i}$ are the mass densities of the liquid and immersions, respectively, and $g$ is the gravitational acceleration. The terminal velocity $u_{s}$ in the upward direction depends crucially on the drag coefficient $C_{D}$ and the effective diameter $D$ of the immersion. In most practical applications, the magnetic field is applied perpendicularly to the main flow as is required for braking or accelerating the flow, measuring the flow velocity, or generating electricity magnetohydrodynamically. For example, in a continuous casting mold, the fluid motion is primarily in the vertical plane, perpendicular to the main field component of an electromagnetic brake. In practice, the most relevant modification to the drag coefficient of an immersion is, thus, typically caused by a magnetic field perpendicular to the motion. Coincidentally, the magnetohydrodynamic modification to the drag coefficient generally is larger for motion perpendicular to the magnetic field compared with that for motion parallel to the magnetic field. ${ }^{[7,8,10-12]}$

The experimental ${ }^{[16]}$ and computational ${ }^{[18]}$ results discussed briefly in the introduction provide confidence that for a bubble motion perpendicular to the magnetic field, the magnetohydrodynamic modifications to the drag coefficient of rigid spheres can be used. Experimental work on rigid spheres ${ }^{[12]}$ reports within the range $17.6 \leq \operatorname{Re} \leq 332$ and $\mathrm{N} \leq 2.5 \times 10^{3}$ a dependence

$$
C_{D}=C_{D 0}(1+0.7 \sqrt{\mathrm{N}})
$$

Here, $C_{D 0}$ is the drag coefficient in the absence of a magnetic field and $\mathrm{N}=\mathrm{Ha}^{2} / \mathrm{Re}=\sigma B_{0}^{2} D / \rho u_{0}$ is the dimensionless interaction parameter, or Stuart number, characterizing the importance of the Lorentz force compared with inertial forces. The Hartmann number $\mathrm{Ha}=B_{0} D \sqrt{\sigma / \rho v}$, with $\sigma, \rho$, and $v$ the respective conductivity, mass density, and kinematic viscosity of the conducting liquid, gives the square root of the ratio between the Lorentz force and viscous forces. Note that both Re and $\mathrm{N}$ are expressed here in terms of the sphere diameter $D$. The relation of Eq. [2] can be refined using the results ${ }^{[19]}$ for $\mathrm{N}, \mathrm{Re} \gg 1$ or the results ${ }^{[8,15]}$ for $\mathrm{Re} \ll 1$, but for most practical purposes, Eq. [2] will suffice.

\section{B. Electromagnetophoretic Force}

In a stagnant fluid in which a homogeneous electrical current density $\mathbf{J}$ exists nonparallel to a constant applied magnetic field $\mathbf{B}$, an equilibrium exists between the Lorentz force and a counteracting pressure force according to $\mathbf{J} \times \mathbf{B}-\nabla p=0$. If the pressure distribution would be unaltered by the presence of an insulating immersion, this would imply a force per unit volume $V$ on the immersion given by $-\frac{1}{V} \int_{V} \nabla p d V=-\mathbf{J} \times \mathbf{B}$, independent of the shape of the object. Near the insulating immersion, however, the current distribution is altered necessarily, resulting in a modified pressure distribution and locally induced fluid flow. The resulting pressure and shear forces can, for a small induced fluid velocity satisfying $\mathrm{Re} \ll 1$, be calculated analytically. ${ }^{[1]}$ Such a calculation yields a force per unit volume given by $-\psi \mathbf{J} \times \mathbf{B}$ where $\psi=3 / 4$. Here, $\psi$ is the shape factor, sometimes also referred to as the form factor or expulsion factor. Under various circumstances, somewhat higher values $0.85 \leq \psi \leq 1.0$ for the shape factor have been reported both numerically ${ }^{[2]}$ and experimentally. ${ }^{[20]}$ This makes the analytical result $\psi=3 / 4$ for $\mathrm{Re} \ll 1$, at least in the literature seem to be a lower bound. A deviation from the analytical result is to be expected when the low-Re fluid flow assumption is not satisfied. Inertial forces can become large if the current becomes large enough to induce a significant fluid velocity or when the object is moving relative to the fluid at a sufficiently high speed. Such a translation velocity can arise because of the electromagnetophoretic force itself or because of an external force like gravity or buoyancy. To the knowledge of the current authors, however, no quantitative results on the electromagnetophoretic force have been reported for the case of objects moving relative to the fluid.

\section{COMPUTATIONAL METHODS}

All computational simulations to be discussed were performed using the commercial computational fluid dynamics software CFX 11.0 (Ansys, Inc., Canonsburg). Because only steady-state simulations were performed, in the next section we will leave out any time dependence. An incompressible Newtonian fluid with a dispersed gas phase has been assumed with the material properties of steel and argon as summarized in Table I. The electrical current density $\mathbf{J}$ is assumed to obey Ohm's law for moving media

$$
\mathbf{J}=\sigma(\mathbf{E}+\mathbf{u} \times \mathbf{B})
$$

with a constant conductivity $\sigma$. The electric field $\mathbf{E} \equiv-\nabla \Phi_{e}$ is solved for, using the fact that in steady state, the current density is divergence free such that a Poisson equation results: $\nabla^{2} \Phi_{e}=\nabla \times(\mathbf{u} \times \mathbf{B})$. In this way, the conservation of charge is ensured automatically. Because the boundary walls are assumed to be insulating, the boundary condition for the electric current is that of zero normal component: $\hat{\mathbf{n}} \times \mathbf{J}=0$.

Table I. Used Material Parameters of Steel and Argon at $1873 \mathrm{~K}\left(1600{ }^{\circ} \mathrm{C}\right)$

\begin{tabular}{lll}
\hline & Steel & \multicolumn{1}{c}{ Argon } \\
\hline Density $\left(\mathrm{kg} / \mathrm{m}^{3}\right)$ & $7 \times 10^{3}$ & 0.3 \\
Dynamic viscosity $\left(\mathrm{kgm}^{-1} \mathrm{~s}^{-1}\right)$ & $7 \times 10^{-3}$ & $8.5 \times 10^{-5}$ \\
Conductivity $\left(\mathrm{Nm}^{-1}\right)$ & $7 \times 10^{5}$ & 0 \\
\hline
\end{tabular}


The magnetic field is calculated as the sum of two parts $\mathbf{B}=\mathbf{B}_{1}+\mathbf{B}_{2}$, one of which follows from solving Ampere's law

$$
\nabla \times \mathbf{B}_{1}=\mu_{0} \mathbf{J}
$$

Added to this is $\mathbf{B}_{2}=-\nabla \Phi_{m}$, the gradient of a magnetic scalar potential $\Phi_{m}$, which is solved for using the fact that the magnetic field is divergence free. This results in yet another Poisson equation: $\nabla^{2} \Phi_{m}=$ $\nabla \times \mathbf{B}_{1}$. The magnetohydrodynamic capabilities of the solver have been validated against analytical solutions.

As a turbulence model, the low Re-number $k-\varepsilon$ model was used, extended with a correction for the magnetohydrodynamic damping of vortices. ${ }^{[21]}$ To account for the flow of the dispersed argon gas, a Eulerian-Eulerian multiphase model was used. The energy equation includes Joule heating.

\section{IMPLEMENTATION}

Simulations were performed for a single insulating sphere first to investigate quantitatively the electromagnetophoretic force. For this purpose, a magnetic field perpendicular to the flow was used such that according to Eq. [3], electric currents exist perpendicular to both the flow and the magnetic field. By varying the strength of an external electric field perpendicular to both the flow and the magnetic field, the magnitude of the electric current around the sphere could be controlled. A sphere with a diameter of $D=1 \mathrm{~mm}$ in a main flow of $u_{0}=1 \mathrm{~cm} / \mathrm{s}$ was used with a constant magnetic field of $B_{0}=0.2 \mathrm{~T}$, yielding a Reynolds number $\mathrm{Re}=10$ and a Hartmann number $\mathrm{Ha}=2$.

A mesh of structured hexahedral elements inward and outward from the sphere was used, with unstructured mesh elements at the center of the sphere and far away from the sphere to fill the rectangular domain. The freeslip domain side walls were positioned at a large enough distance to have a vanishingly small effect of the sphere on the flow near these walls. The dimensions of the rectangular domain were chosen to be 10,15 , and 30 sphere diameters $D$ in the direction parallel to the electric field, the magnetic field, and the main flow, respectively. The mesh size was fine enough to resolve the thin Hartmann layer developing on the surface of the sphere. For this purpose, the first mesh layer in the fluid was much smaller than $D / \mathrm{Ha}_{D}=\sqrt{\rho v / \sigma B_{0}^{2}}$.

Finally, simulations in a realistic continuous caster geometry (Table II) were performed of which the results

Table II. Parameters Used in the Continuous Casting Simulations

\begin{tabular}{ll}
\hline Property & \multicolumn{1}{c}{ Quantity } \\
\hline SEN diameter & $7.5 \mathrm{~cm}$ \\
SEN length & $40 \mathrm{~cm}$ \\
Casting speed & $0.03 \mathrm{~m} / \mathrm{s}$ \\
Magnetic field strength & $<0.3 \mathrm{~T}$ \\
Steel temperature & $1873 \mathrm{~K}\left(1600^{\circ} \mathrm{C}\right)$ \\
\hline
\end{tabular}

for the submerged entry nozzle are reported here. The magnetic field is modeled to that of an FC-II mold. The field is assumed to be constant in the direction of the width of the caster, but it shows a strong variation in the vertical direction as shown in Figure 4(b). Far into the entry nozzle the magnetic field remains significant.

\section{RESULTS}

In this section, we discuss first the magnetohydrodynamic increase in the drag coefficient on the terminal rise velocity of both rigid inclusions and bubbles. Next, simulations are presented of a single rigid sphere with a significant velocity difference between the sphere and the main flow. From these simulations, quantitative expressions are obtained for the electromagnetophoretic form factor. Finally, the newly obtained expression for the electromagnetophoretic force is implemented in a multiphase model for argon and steel in a continuous casting mold, for which some interesting observations regarding the submerged entry nozzle are presented.

\section{A. Magnetohydrodynamically Modified Drag}

The impact of a modified drag coefficient on the terminal rise velocity of spheres and bubbles is displayed in Figure 1. The curves were obtained in an iterative manner from Eq. [1], using the correlations for the drag coefficient of rigid spheres as reported in Reference 22, together with the magnetohydrodynamic correction of Eq. [2]. Reference 22 also contains a fit to a large body of data on the terminal rise velocity of bubbles in the ellipsoidal regime, which has been used to obtain the second curve in the figure. Because the results for the terminal rise velocity of bubbles in the presence of a magnetic field are a bit more speculative, these curves are displayed as dashed lines and have been obtained in a noniterative way from Eq. [5].

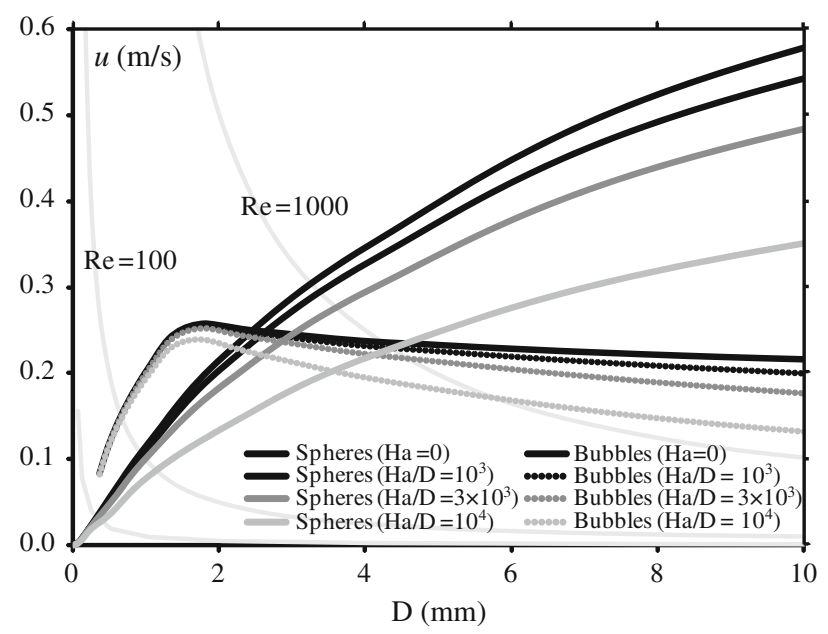

Fig. 1-The terminal rise velocity obtained for spheres and argon bubbles in liquid steel, in the presence of a perpendicular magnetic field. For the material properties of liquid steel as given in Table I, $\mathrm{Ha} / D=1000$ corresponds to, e.g., a sphere with a diameter of $D=1 \mathrm{~mm}$ and a magnetic field of $B_{0}=0.1 \mathrm{~T}$. 
For Reynolds numbers that are not too small, the magnetohydrodynamic modification of the drag becomes significant when the Lorentz force becomes comparable in size to inertial forces, i.e., when $\mathrm{N}=\sigma B_{0}^{2} D / \rho u_{0}$ differs appreciably from zero. Spherical particles rising under the influence of buoyancy, from Eq. [1], have a terminal rise velocity $u_{s} \propto \sqrt{D / C_{D}}$, which implies that for high $\operatorname{Re} \geq 10^{3}$, for which the drag coefficient of rigid spheres is approximately constant, $u_{S}$ is proportional to $\sqrt{D}$. From Eq. [2], the fractional increase $\left(C_{D}-C_{D 0}\right) / C_{D 0}$ in drag coefficient is in this case proportional to $\sqrt{\mathrm{N}} \propto D^{\frac{1}{4}}$. Because large spherical particles can, under the influence of a body force like buoyancy, obtain Reynolds numbers $\operatorname{Re} \geq 10^{3}$, in most circumstances we expect a slight positive dependence of the drag coefficient on the particle size. For $\mathrm{Re} \ll 1$, the drag coefficient attains the Stokes value of $C_{D}=24 / \operatorname{Re} \propto 1 / u_{0} D$ such that $u_{s} \propto D^{2}$ and the fractional increase in drag coefficient becomes proportional to $\sqrt{\mathrm{N}} \propto D^{-\frac{1}{2}}$. For the small particles, moving in the Stokes range $\operatorname{Re} \ll 1$, the drag coefficient thus shows a negative dependence on the particle size. For small bubbles, this same conclusion holds more or less. For larger bubbles with $D>2 \mathrm{~mm}$, as can be observed from Figure 1, the terminal rise velocity $u_{s} \propto \sqrt{D / C_{D 0}}$ approximately stagnates, which implies that for large bubbles, the drag coefficient becomes more or less proportional to the bubble diameter $D$. The terminal rise velocity for bubbles with $D>2 \mathrm{~mm}$ in the presence of a perpendicular magnetic field can, thus, be approximated by

$$
u_{s} \approx \frac{u_{s 0}}{\sqrt{1+0.7 \sqrt{\mathrm{N}}}}
$$

which for the important regime $\sqrt{\mathrm{N}} \gg 1$ can be approximated by $u_{s} \approx 1.2 u_{s 0} \mathrm{~N}^{-\frac{1}{4}}$, and for the somewhat less interesting regime $\sqrt{\mathrm{N}} \ll 1$, by $u_{s} \approx u_{s 0}\left(1-0.35 \mathrm{~N}^{\frac{1}{4}}\right)$. Because of the double square root in Eq. [5], the terminal rise velocity of bubbles is, thus, fairly insensitive to $N$ but more significantly dependent on $\mathrm{Ha}$ and $\mathrm{Re}$.

\section{B. Electromagnetophoretic Force}

Simulations of a single rigid sphere in the presence of an electric and magnetic field perpendicular to each other and to the flow have been performed under the conditions described in Section IV. To investigate the magnitude of the electromagnetophoretic force, the total force on the sphere has to be compensated for the viscous and magnetohydrodynamic drag. For this purpose, a reference simulation has been performed with an electric field $\mathbf{E}_{0}=-\mathbf{u}_{0} \times \mathbf{B}_{0}$ to cancel the formation of currents far from the sphere. Here, subscript 0 denotes a constant far-field value for the corresponding quantity. With the value of the drag force thus obtained subtracted, the remaining force could be attributed to the electromagnetophoretic force. The resulting net force was indeed found to be in the direction of $-\mathbf{J}_{0} \times \mathbf{B}_{0}$ and the magnitude per unit volume in units of $J_{0} B_{0}$ is depicted in Figure 2. It can be noted that this force, up to quite high values of the current density, is described

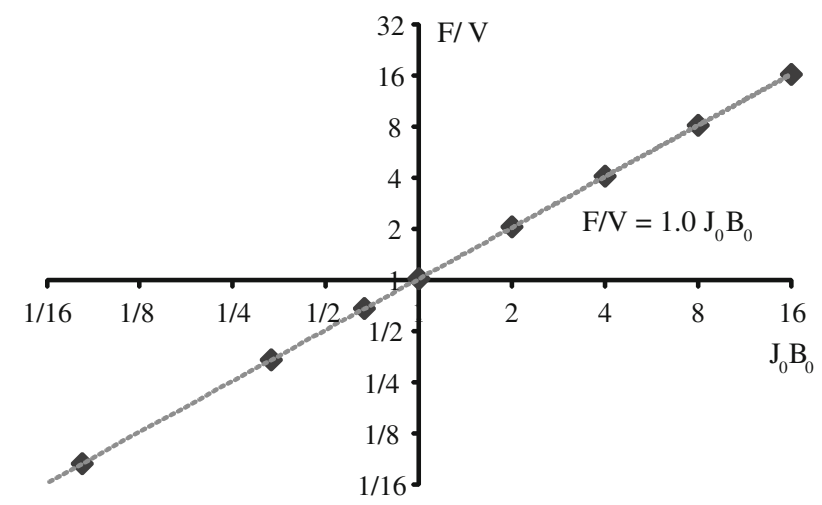

Fig. 2-Electromagnetophoretic force per unit volume $-\psi \mathbf{J} \times \mathbf{B}$ as a function of $J B$, both in units of $\sigma u_{0} B_{0}^{2}=140 \mathrm{Nm}^{-3}$. These computational results correspond to $\mathrm{Re}=10, \mathrm{Ha}=2$ for the case that $\mathbf{J}$ is perpendicular to both $\mathbf{u}$ and $\mathbf{B}$. Simulations with $\mathbf{J}$ parallel to u showed similar results.

very accurately by a linear relationship. The resulting shape factor was found to be approximately equal for both investigated magnetic field directions and close to $\psi=1$. The electric current distributions for various values of the electric field are shown in Figure 3. A detailed analysis of the flow, electric charge, and the electromagnetic fields around an insulating sphere, in the case of a vanishing far-field current, can be found in Reference 15.

\section{Submerged Entry Nozzle (SEN)}

For the electromagnetophoretic force to be of significance, the force density $J B$ has to be comparable in size with that of the other forces acting on the gas phase. In particular, it has to be comparable with buoyancy, which is, using the data in Table I, of the order of $\rho g \approx 7 \times 10^{4} \mathrm{Nm}^{-3}$. From steady $=$ state simulations, this was observed to be the case in fairly limited localized regions within the casting mold, with the specifics depending crucially on the magnetic field distribution and operational parameters like the SEN port angle. Ultimately, the details will depend on the transient behavior of the jets emerging from the SEN as well. Inside the SEN (Figure 4), however, the situation is somewhat more clear cut. Although the flow is heavily turbulent, the main velocity component will always be directed downward and will go to zero near the SEN walls. Based on these universal characteristics, some general conclusions can be drawn concerning the effect of the electromagnetophoretic force on the gas phase.

The downward fluid motion inside the SEN perpendicular to the magnetic field induces an electrical current according to Ohm's law, Eq. [3]. The electric field set up by the charge accumulating at the insulating pipe wall forces the electric currents to recirculate via the flow boundary layer as shown in Figure 5. These electrical currents brake the fluid motion in the vertical direction and thus accelerate gas downwards. Looking at the vector plot of the electromagnetophoretic force in Figure 5, this is indeed confirmed by the performed computational simulations. What can also 


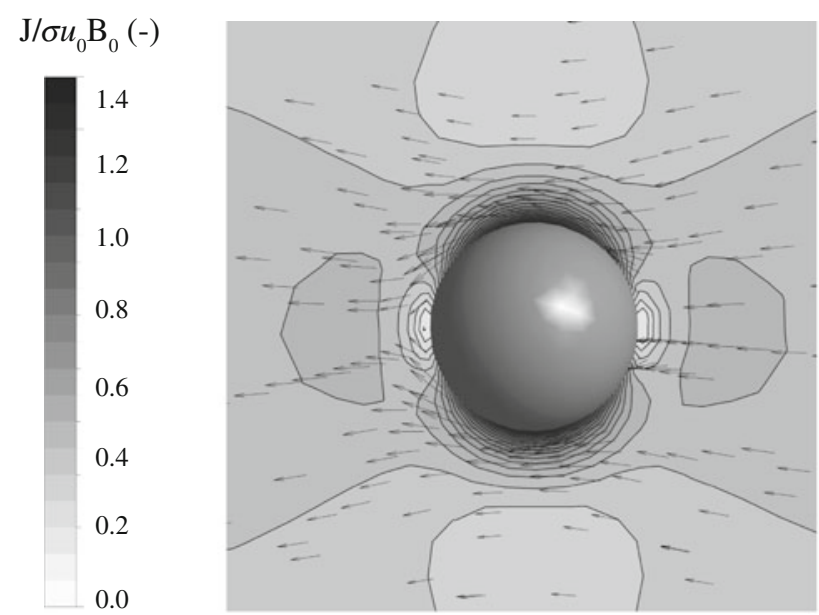

(a)

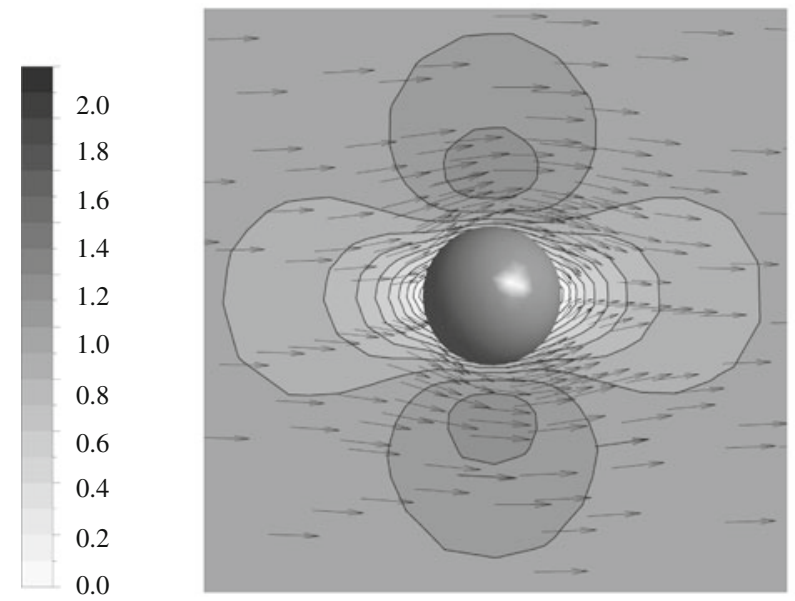

(c)

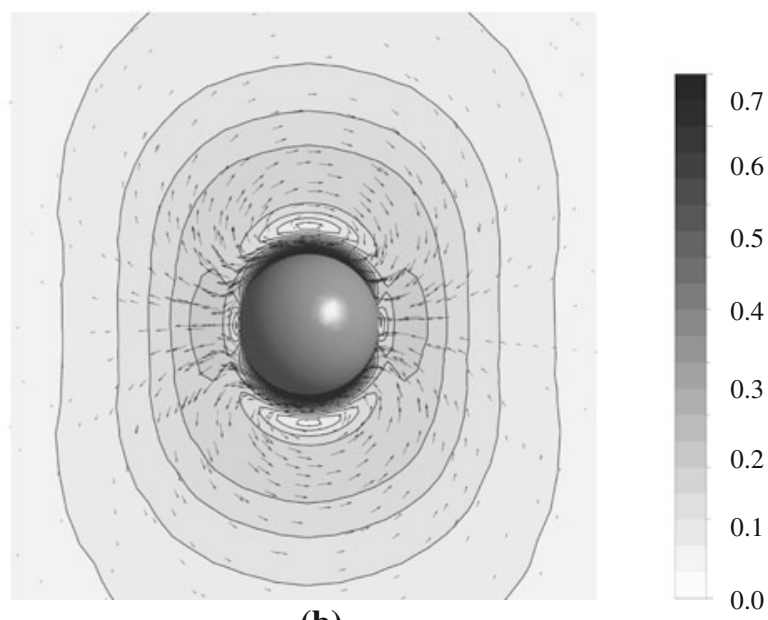

(b)

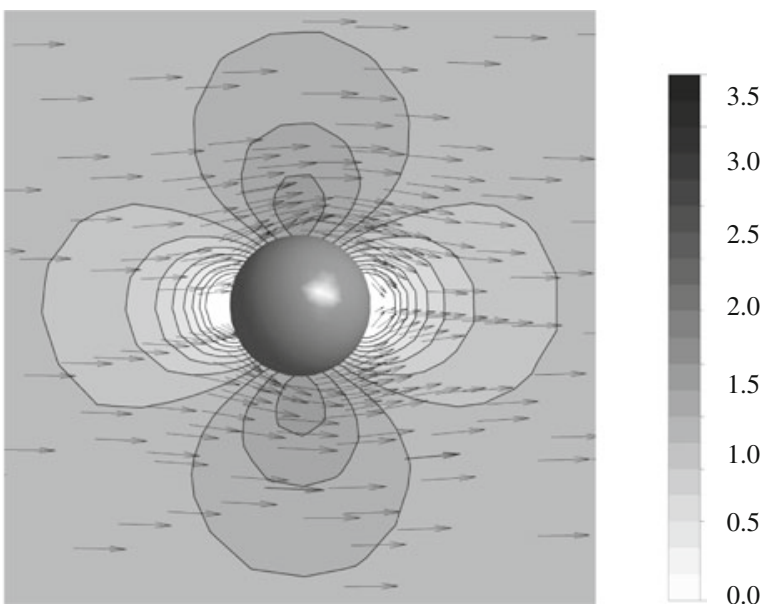

(d)

Fig. 3-The current density $\mathbf{J}$ in the vicinity of an insulating sphere for $\mathrm{Re}=10, \mathrm{Ha}=2$, and $\mathbf{N}=0.4$. Various values of the electric field $\mathbf{E}_{0}$ perpendicular to both the main flow velocity $\mathbf{u}_{0}$ and the magnetic field $\mathbf{B}_{0}$ are used such that different values for the far-field current density $\mathbf{J}_{0}$ result. The images apply to the situation with (a) $\mathbf{E}_{0}=-\frac{3}{2} \mathbf{u}_{0} \times \mathbf{B}_{0}$, (b) $\mathbf{E}_{0}=-\mathbf{u}_{0} \times \mathbf{B}_{0},(c) \mathbf{E}_{0}=0$, and (d) $\mathbf{E}_{0}=\mathbf{u}_{0} \times \mathbf{B}_{0}$ resulting in a far field current $\mathbf{J}_{0}=-\frac{1}{2} \mathbf{u}_{0} \times \mathbf{B}_{0}, 0, \mathbf{u}_{0} \times \mathbf{B}_{0}$ and $2 \mathbf{u}_{0} \times \mathbf{B}_{0}$, respectively.

be determined from these computational results, however, is the presence of a radial component of the electromagnetophoretic force. Such a radial component must be necessarily caused by axial electric currents, which can indeed be observed from the simulations. Especially close to the nozzle walls, as shown in Figure 5(b), these vertical electrical currents are significant. The currents are directed in opposite directions at opposite side walls. What can also be observed is a change in the direction of these currents at a vertical position, more or less coinciding with the local maximum in the magnetic field.

The reason for the existence of these currents can be found from Ampere's Law. Taking the curl of Eq. [4], using the mathematical identity $\nabla \times(\nabla \times \mathbf{B})=$ $\nabla(\nabla \cdot \mathbf{B})-\nabla^{2} \mathbf{B}$ and the fact that $\nabla \cdot \mathbf{B}=0$ one obtains

$$
\nabla \times \mathbf{J}=-\frac{1}{\mu_{0}} \nabla^{2} \mathbf{B}
$$

Therefore, a curl in the current density $\mathbf{J}$ arises whenever the Laplacian of the magnetic field is non-zero. In the coordinates of Figure 4, the magnetic field only has a component in the $y$-direction $\hat{\mathbf{y}}$ and varies only as a function of $z$, i.e., $\mathbf{B}=B(z) \hat{\mathbf{y}}$. In this case, Eq. [6] yields

$$
(\nabla \times \mathbf{J})_{y}=-\frac{1}{\mu_{0}} \frac{d^{2} B}{d z^{2}}
$$

Depending on the second derivative of the magnetic field to the axial coordinate $z$, the currents, therefore, curl clockwise or counterclockwise around the magnetic field.

Because the magnetic field for high $z$ is a convex function of $z$ satisfying $d^{2} B / d z^{2}<0$, as observed from Figure 4, we infer from Eq. [7] that the curl of the electric current is in the positive $y$-direction, which is in accordance with the results from the simulations of Figure 5. After some distance in the negative $z$-direction, the magnetic field becomes a concave function of $z$, i.e., it attains $d^{2} B / d z^{2}>0$, and the curl of the current changes direction. This can indeed be observed from Figure 5. In the first part of the SEN, for 


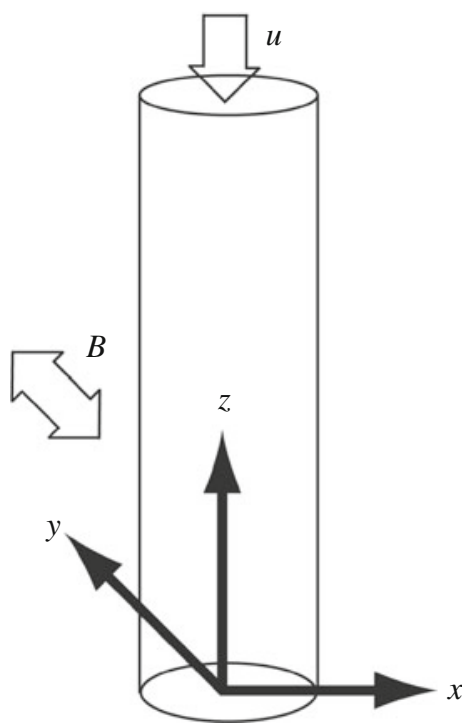

(a)

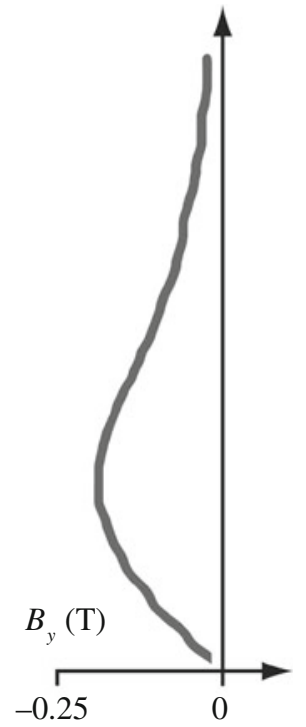

(b)
Fig. 4-A schematic overview of $(a)$ the geometry and coordinates of the SEN and $(b)$ the magnetic field strength used in the simulations.
0

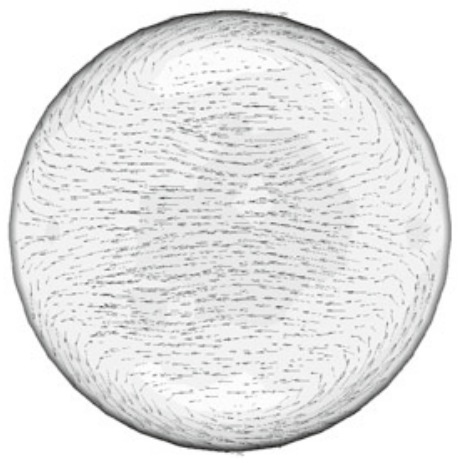

(a)

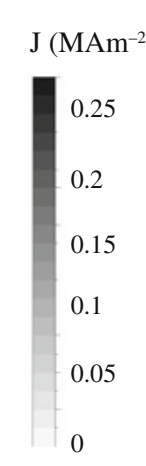

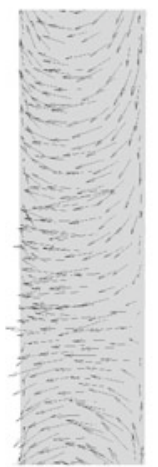

(b)
Fig. 5-(a) The current density $\mathbf{J}\left(\mathrm{MAm}^{-2}\right)$ in a horizontal cross section of the SEN and $(b)$ the direction of the currents in a vertical cross section ( $x z$-plane) perpendicular to the magnetic field.

large $z$, the electrical currents are, therefore, in the negative $z$-direction for positive $x$ and in the positive $z$-direction for negative $x$. With the magnetic field being directed in the negative $y$-direction, we observe that the associated electromagnetophoretic force is in the positive $x$-direction for positive $x$ and in the negative $x$-direction for negative $x$, i.e., directed outward from the $y z$-plane. This same observation is found in Figures 5 and 6 . Now for the effect of these axial currents and the associated electromagnetophoretic force, we examine the distribution of gas as a function of the distance along the pipe as shown in Figures 6 and 7. The electromagnetophoretic force, being directed away from the $y z$-plane, can be observed to alter the distribution of gas significantly. The gas enters the pipe homogeneously distributed, but under the influence of

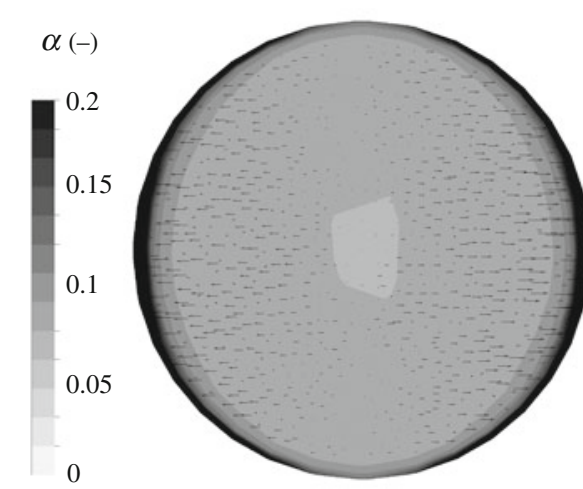

(a)

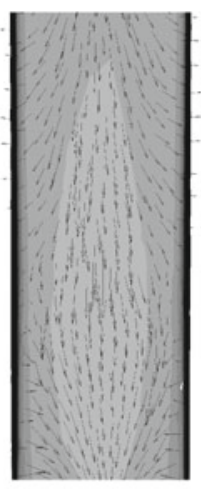

(b)
Fig. 6 - (a) The argon volume fraction $\alpha(-)$ and the direction of the electromagnetophoretic force $-\mathbf{J} \times \mathbf{B}$ in a horizontal cross section and $(b)$ in a vertical cross section ( $x z$-plane) perpendicular to the magnetic field.

this force, more and more gas moves toward the sides of the SEN. After some distance, the direction of the electric currents reverses at the location at which $d^{2} B / d z^{2}$ changes sign and the gas was observed to move slowly away from the sides again. This finding is in sharp contrast with simulations in which the electromagnetophoretic force was absent, in which case the gas distribution was observed to remain more or less homogeneously distributed.

\section{CONCLUSIONS}

Even though insulating objects like nonmetallic particles or bubbles immersed in a conducting liquid do not experience a Lorentz force, various significant magnetohydrodynamic effects can occur indirectly through the surrounding conducting fluid. The drag coefficient of spherical inclusions moving perpendicular to a magnetic field fractionally increases approximately proportional to the square root of the interaction parameter $\mathrm{N}$, and a similar correction for bubbles constitutes an improvement in the modeling of the drag force as well. For a relevant choice of parameters, the increase in drag coefficient was shown to result in a significant decrease in terminal rise velocity. This finding most likely has a negative effect on the probability that inclusions and bubbles escape at the meniscus of a continuous caster. The electromagnetophoretic force on insulating inclusions and bubbles acts opposite to the Lorentz force on the conducting liquid, and therefore, it acts mostly in the direction of the fluid flow. Its effect on the gas distribution was investigated for an inhomogeneous magnetic field in a submerged entry nozzle of a continuous caster. Depending on the shape of the magnetic field, concave or convex as a function of the vertical coordinate, the electromagnetophoretic force was shown to be directed inward or outward, respectively. In the latter case, the gas was found to distribute itself with an increased concentration near the nozzle side walls. Depending on the positive or negative effects 


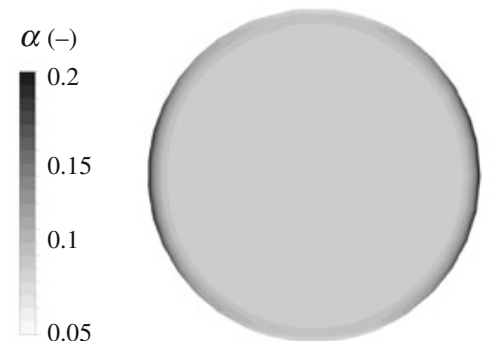

(a)

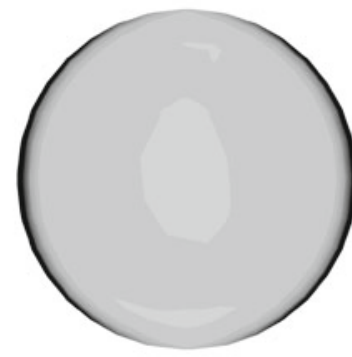

(b)

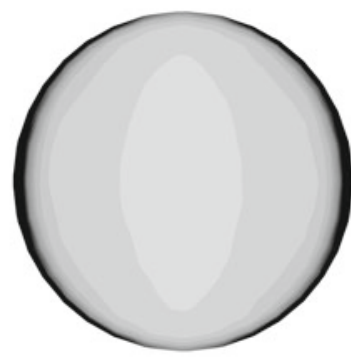

(c)

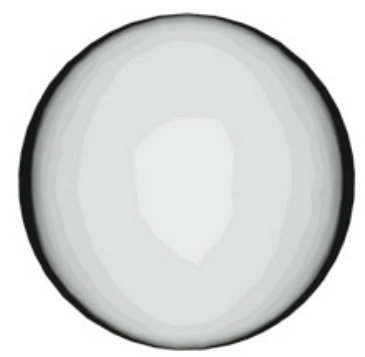

(d)

Fig. 7-The gas volume fraction distribution in a cross section of the SEN at 20-cm intervals in the negative $z$-direction.

of an inhomogeneous gas distribution on e.g., clogging behavior, the specific shape of the magnetic field can be designed either to increase or decrease the amount of gas near the side walls.

\section{OPEN ACCESS}

This article is distributed under the terms of the Creative Commons Attribution Noncommercial License which permits any noncommercial use, distribution, and reproduction in any medium, provided the original author(s) and source are credited.

\section{REFERENCES}

1. D. Leenov and A. Kolin: J. Chem. Ph., 1953, vol. 22, pp. 683-88.

2. T.T. Natarajan and N El-Kaddah: Metall. Mater. Trans. B, 2002, vol. 33B, pp. $775-85$.

3. D. Shu, B.-D. Sun, J. Wang, T.-X. Li, Z.-M. Xu, and Y.-H. Zhou: Metall. Mater. Trans. B, 2000, vol. 31B, pp. 1527-33.

4. V.M. Bazilevskii, V.M. Okunev, I.L. Povkh, V.A. Popov, V.A. Smirnov, and B.V. Chekin: Magnitnaya Gidrodinamika, 1970, vol. 6 , pp. 155-57.
5. P. Marty and A. Alemany: Proc. IUTAM Symp. The Metals Soc., 1982, p. 245

6. B. Li and F. Tsukihashi: ISIJ Int., 2003, vol. 43, pp. 923-31.

7. W. Chester: J. Fluid Mech., 1957, vol. 3, p. 304.

8. K. Gotoh: J. Phys. Soc. Jpn., 1960, vol. 15, pp. 696-705.

9. J.R. Reitz and L.L. Foldy: J. Fluid Mech., 1961, vol. 11, pp. 133-42.

10. G. Yonas: J. Fluid Mech., 1967, vol. 30 (4), pp. 813-21.

11. G.G. Branover, N.M. Slyusarev, and A.B. Tsinober: Magnitnaya Gidrodinamika, 1966, vol. 2, pp. 149-50.

12. Kh.E. Kalis, N.M. Slyusarev, A.B. Tsinober, and A.G. Shtern: Magnitnaya Gidrodinamika, 1966, vol. 2, pp. 152-53.

13. T.V.S. Sekhar, R. Sivakumar, and T.V.R. Ravi Kumar: Fluid Dyn. Res., 2005, vol. 37, pp. 357-73.

14. T.V.S. Sekhar, T.V.R. Ravikumar, and H. Kumar: Computational Mechanics, 2003, vol. 31, pp. 437-44.

15. J.W. Haverkort and T.W.J. Peeters: Magnetohydrodynamics, 2009, vol. 45, pp. 111-26.

16. S. Eckert, G. Gerbeth, and O. Lielausi: Int. J. Multiphase Flow, 2006, vol. 26, pp. 67-82.

17. C. Zhang, S. Eckert, and G. Gerbeth: Int. J. Multiphase Flow, 2005, vol. 31, pp. 824-42.

18. K. Takatani: ISIJ Int., 2007, vol. 47, pp. 545-51.

19. J.C.R. Hunt: Magnitnaya Gidrodinamika, 1970, vol. 6, pp. 35-38.

20. E.I. Dobychin and V.I. Popov: Magnitnaya Gidrodinamika, 1979, vol. 6 (3), pp. 74-78.

21. S. Kenjereš and K. Hanjalić: Int. J. Heat Fluid Flow, 2000, vol. 21, pp. 329-37.

22. J.R. Grace, R. Clift, and M.E. Weber: Bubbles Drops and Particles. Academic Press, Inc., 1978. 